\title{
An Effective Mammogram Classification Using Hot Based Tree And Hot Based Cnn
}

\author{
Girija O K ${ }^{\mathrm{a}}$, 2 Sudheep Elayidom ${ }^{\mathrm{b}}$ \\ ${ }^{a}$ Pursuing Ph.D. in the Division of Computer Science and Technology, School of Engineering, CUSAT, Kochi, INDIA \\ ${ }^{\mathrm{b}}$ Professor in the Division of Computer Science and Technology, School of Engineering, CUSAT, Kochi, INDIA
}

Article History: Received: 10 November 2020; Revised 12 January 2021 Accepted: 27 January 2021; Published online: 5 April 2021

\begin{abstract}
Breast cancer is the subsequent leading cause of cancer-related deceases among women. Initial exposure stimulates enhanced visualization and saves survives. These days, the exact grouping classification of breast cancer images is a difficult errand. There are much research works delivering various strategies and algorithms for this specific errand of medical image processing. To build up an exact characterization, this paper presents a viable classification of mammogram images utilizing HOT based classification tree and HOT based convolutional neural network $(\mathrm{CNN})$. The input breast image is at first taken from the database and pre-processed by RGB to grayscale conversion and normalization methodology. In this way Histogram of Oriented Texture (HOT) Descriptor is extorted from the pre-processed images. At long last images are classified as typical or irregular utilizing HOT based classification tree and HOT based CNN. The exploratory results show that the introduced method outperforms the existing strategies concerning various performance assessments like accuracy, sensitivity, specificity, mean absolute error, AUC score, kappa statistics, and Root mean square error
\end{abstract}

Keywords: RGB to gray conversion, Gabor filter, HOG, HOT descriptor, Classification tree, convolutional neural network.

\section{Introduction}

Breast cancer has convert the maximum ordinarily analyzed malignancy in ladies, especially in ladies more seasoned than 40 [1]. As shown by the latest bits of knowledge from the International Agency for Research on Cancer (IARC), which is the related establishment of the World Health Organization (WHO), the overall steady number of ladies with bosom tumors outperforms 2, 080, 000, speaking to $24.2 \%$ among ladies with malignant growths [2]. In the past 20 years, the pace of breast cancer has been continually growing, which sabotages ladies' prosperity, spirits even live truly and destroy different families in the public arena [3] [4]. Fortunately, early discovery benefits the endurance pace of ladies with breast cancer. According to long stretch clinical experience, bosom malignant growth can be forestalled or even restored by viable preventive treatment $[5,6]$. Therefore, setting up indicative models for breast cancer growth is of essential criticalness. Early ID and therapy can reduce the bosom malignancy demise rate essentially. Incredibly, in breast cancer, the signs are unnoticeable and vary in appearance toward the early phases [7].

To envision the inside breast structures a low segment $\mathrm{x}$-light emission breasts are completed, this technique in clinical terms is known as Mammography. It is one of the most sensible strategies to recognize breast disease [8]. Mammograms today open the breast to much lower segments of radiation differentiated and devices used previously. Lately it has been confirmed to be one of the most reliable instruments for screening and a critical system for the early revelation of breast disease [9]. With the improvement of imaging contraption and AI innovation, progressed histopathology picture investigation transforms into a promising method to manage a solid and cost-beneficial disease finding. Particularly for constant breast malignant growth, given the customary data that cancer-causing cells get past the basement film of ductulo-lobular structures and enter neighboring tissues the component of meddling [10], various calculations were proposed to assemble breast histopathology pictures using cores' morphology and spatial-circulation highlights [11, 12].

Various scientists have utilized area and overall surface information from the mammograms using strategies, for example, neighborhood paired example [13], [14] dark level co-event grids [15] and shape properties, and so forth Others have mishandled multi-goal systems, for instance, wavelets [16], curve lets for include extraction [17], [18], and orders of mammograms. Deep Convolutional Neural Network (DCNN) is a feed-forward profound learning plan that used to deal with order issues in the field of PC vision for a serious long time. DCNN deals with the order issue by removing the highlights through various convolution channels. The presentation of the off-rack classifiers like Support Vector Machine (SVM), arbitrary woods are commonly affected by the idea of preparing information and look at the issues through the highlights considered [19]. The contribution of this work is

- $\quad$ Effective arrangement of mammogram pictures using HOT based tree 
- $\quad$ HOT based convolutional neural organization $(\mathrm{CNN})$

In an association, Section-2 related work the connected functions concerning the proposed procedure. In area 3 , a concise discussion about the proposed system is introduced, segment 4 looks at the primer results, and segment 5 wraps up the paper.

\section{Related Work}

Zhiqiong Wang et al. [20] presented a breast Computer-Aided Diagnosis procedure reliant on component grouping with CNN thoughtful highlights. At first, they proposed a mass acknowledgment method reliant on CNN thoughtful highlights and Unsupervised Extreme Learning Machine (US-ELM) grouping. Next, we gather a rundown include set combining deep highlights, external highlights, and depth highlights. From that point onward, an ELM classifier was made using the combined list of capabilities to group genial and perilous breast masses. Expansive investigations display the precision and proficiency of their proposed mass identification and breast malignant growth characterization methodology.

Hasan Nasir Khan et al. [21] proposed a Multi-View Feature Fusion (MVFF) based CADx scheme by a component combination methodology of four viewpoints for portrayal of a mammogram. The all-out CADx gadget comprises three phases, the chief stage can describe mammograms as unusual or ordinary, and the subsequent stage was about grouping of mass or calcification and in the last phase portrayal of risky or liberal order was performed. Convolutional Neural Network (CNN) based component abstraction models work concerning every observation freely. These removed highlights were joined into single last layer for the outrageous gauge.

Tianyu Shen et al. [22] proposed a blended oversight guided procedure and a lingering helped order U-Net model (ResCU-Net) for joint division and considerate harmful arrangement. By coupling the solid management as division cover and frail oversight as the kindhearted harmful mark through a straightforward comment strategy, our method profitably sections tumor locales all the while predicting a discriminative guide for perceiving the benevolent threatening kinds of tumors. Our framework, ResCU-Net, broadens U-Net by combining the leftover module and the SegNet engineering to misuse staggered data for achieving improved tissue ID.

Monjoy Saha and Chandan Chakraborty [23] proposed a profound learning-based HER2 profound neural framework (Her2Net) to understand this subject. The convolutional and deconvolutional parts of the proposed Her2Net framework included principally of various layers, and trapezoidal long transient memory (TLSTM). A completely associated layer and a softmax layer were in like manner used for order and blunder assessment. Finally, HER2 scores were resolved reliant on the grouping results. The key responsibility of their proposed Her2Net framework fuses the execution of TLSTM and a profound knowledge system for cell film and core recognition, division, and grouping, and HER2 counting.

Yuqian Li et al. [24] proposed the movement in profound learning convolutional neural frameworks (CNNs) for histology picture examination. The characterization of breast malignancy histology pictures into ordinary, kind, and destructive sub-classes was related to cells' thickness, alterability, and relationship alongside by and large tissue structure and morphology. In view of this, they extricated both more modest and greater size patches from histology pictures, including cell-level and tissue-level highlights, correspondingly. In any case, some analyzed cell-level fixes don't contain enough information that organizes the picture tag. Thusly, they proposed a patches' screening procedure subject to the grouping calculation and CNN to pick more discriminative patches.

\section{Proposed Methodology}

A HOT based classification tree and HOT based CNN for the capable breast cancer classification is presented. The proposed strategy methodology is portrayed as the input breast image is pre-processed by RGB to grayscale transformation and normalization systems. In this way HOT Descriptor is extracted from the preprocessed images. At last, images are named typical or irregular utilizing HOT based classification tree and HOT based CNN. The flow illustration of the presented procedure is described in figure 1. 


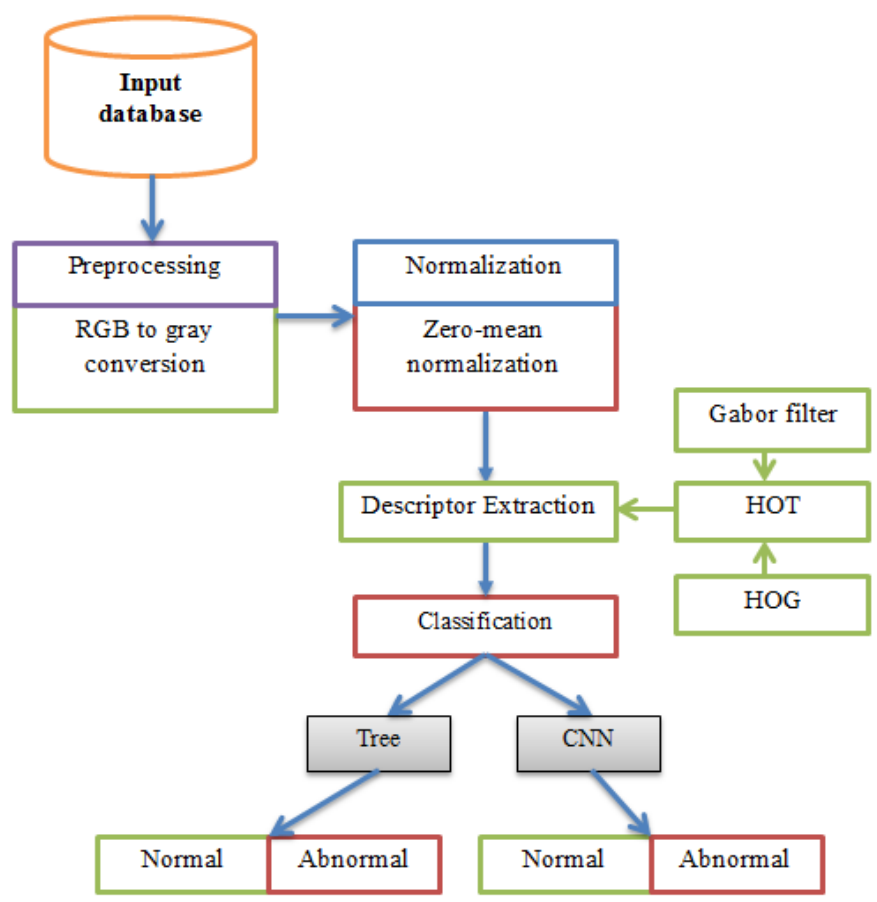

Figure 1: Block illustration of the proposed methodology

\subsection{Preprocessing}

\subsubsection{RGB to gray conversion}

Transformation of a color image into a grayscale image comprehensive of significant features is a Transformation of a shading image into a grayscale image exhaustive of critical features is a convoluted procedure. As such, all color input and the grayscale output are situated somewhere in the range of 0 and 255 . On this scale, 0 characterize to dark and 255 characterize to white. The color image to grayscale image conversion is given in condition (1)

$$
G_{s}=0.201 * R_{e d}+0.701 * G_{r e}+0.070 * B_{l u}
$$

It is accepted that $R_{e d}, G_{r e}$, and $B_{l u}$ denote a direct symbol of the red, green, and blue channels individually.

\subsubsection{Zero-mean normalization}

The principal reason for this is to diminish the impedance of medical images. Zero-mean standardization is the standardization of data for the mean and standard deviation of the crude information. The handled information changes with the standard ordinary dispersion, that is, the mean is 0 , the standard deviation is 1 , and the transformation work is as appeared in (2),

$$
s^{*}=\frac{s-\mu}{\sigma}
$$

Where, $\mu$ signifies the mean of trial information, $\sigma$ signifies the standard deviation of trial information.

\subsection{The Histogram of Oriented Texture (HOT) Descriptor}

Here, HOT descriptor is determined. Initially, image inclination and direction is computed from the cells and square segments. The incline of an image $I$ in horizontal and vertical information for a pixel location $(s, t)$ is calculated as,

$$
\begin{aligned}
& \hat{d} s=\hat{I}(s+1, t)-\hat{I}(s-1, t) \\
& d y=I(s, t+1)-I(s, t-1)
\end{aligned}
$$

For every pixel $I(s, t)$ the slope size $m(s, t)$ and direction $\theta(s, t)$ are determined as underneath, 


$$
\begin{gathered}
\hat{m}(s, t)=\sqrt{\hat{d} s^{2}+\hat{d} t^{2}} \\
\& \theta(s, t)=\tan ^{-1}\left(\frac{d t}{d s}\right)
\end{gathered}
$$

The histogram of directions $H D(a)_{i}$ inside $i^{\text {th }}$ cell is calculated as,

$$
H D(a)_{i}=H D(a)_{i}+\hat{m}(s, t)
$$

$$
\hat{m}(s, t) \in \text { cell }_{i}
$$

Here, $a=1,2,3 \ldots A$ and $i=1,2,3 \ldots . . c$. The histogram of $j^{\text {th }}$ the wedge $H D(a)_{j}$ is gotten by coordinating Histogram of Cells ( $H C s$ ) inside this square as beneath,

$$
H D_{j}=H C_{1}\left\|H C_{2}\right\| \ldots . . \mid H C_{l \times l}
$$

Here,, $\mid$ indicates histograms link into a vector. The vector $H D_{j}$ is at long last standardized as beneath to acquire $H D_{j}$.

$$
\bar{n} H D_{j}=\frac{H D_{j}}{\sqrt{\left\|H D_{j}\right\|^{2}+\bar{e}^{2}}}
$$

Here, $\bar{e}$ denotes a little consistent to keep away from the issue of division by zero. HOG is acquired by incorporating standardized histograms of all squares as beneath.

$$
\overline{H O G}=\bar{n} H D_{1}\left\|\bar{n} H D_{2}\right\| \ldots . . \bar{n} H D_{j}\|\ldots .\| \bar{n} H D_{\bar{n}}
$$

Here, $\bar{n}$ denotes a quantity of potential squares in image. As such, a Gabor filter work is portrayed as beneath,

$$
\bar{G}(s, t, \hat{\theta}, \mu, \sigma)=\frac{1}{2 \pi \sigma^{2}} \exp \left\{-\frac{s^{2}+t^{2}}{2 \sigma^{2}}\right\} \exp [2 \pi i(\mu s \cos \hat{\theta}+\mu t \sin \hat{\theta})]
$$

Here, $\mu$ denotes the recurrence of the sinusoidal wave, $\hat{\theta}$ denotes a direction, and $\sigma$ denotes a standard deviation. Presently, surface component of image $(\hat{I})$ is determined by the genuine portion of a Gabor channel manage an account with four different directions and a static scale. Gabor size $\hat{m}(s, t)_{\text {Gabor }}$, and Gabor direction $\hat{\theta}(s, t)_{\text {Gabor }}$, the response of every pixel $(s, t)$ are determined as,

$$
\hat{m}_{\text {Gabor }}(s, t)=\min (\hat{I}(s, t) * \bar{G}(s, t, \hat{\theta}, \mu, \sigma))
$$

Furthermore, $\quad \hat{\theta}_{\text {Gabor }}(s, t)=\arg \min (\hat{I}(s, t) * G(s, t, \hat{\theta}, \mu, \sigma))$

Where, $*$ implies the difficulty activity, the direction $\hat{\theta}_{t}$ is determined as underneath:

$$
\hat{\theta}=\frac{\pi(t-1)}{4}, t=1,2, \ldots .4
$$

At last, ideal boundaries of the HOT descriptor are chosen by experimentation. For that, the direction range $\left(0^{\circ}-180^{\circ}\right)$ is quantized into 4 cases only. 


\subsection{Classification using HOT based classification tree}

The introduced tree-based classification of benign and malignant afterwards finding and dividing the speculated abnormal areas. The beginning stage is the automated recognition of a micro-calcification cluster as ROIs. These ROIs area unit precisely representing image as a binary form, wherever ' 1 ' and ' 0 ' of binary microcalcification for each component within a picture.

\section{$>$ Binarization}

we tend to binaries the ROIs that extracts small chance areas. Binarization is denoted as,

$$
P\left(s_{i}, t_{j}\right)= \begin{cases}1, & \text { if } P\left(s_{i}, t_{j}\right) \geq T h r \\ 0, & \text { otherwise }\end{cases}
$$

In condition (16), the edge is denoted as Thr. Set Thr to make a binary image that's harmony among eliminating noise and retaining important image info that may be required for characterization. Given the trials, we have a tendency to see that 0.27 is that the best $T h r$ worth relating to obtaining simply the many segmented the micro-calcifications.

\section{$>\quad$ Isolating associated mechanisms and group of the node}

Node-Identity is allocated steadily for altogether nodes, though the left and right children are null as there are no associations among the nodes at this stage.

\section{Distance-map calculation}

Determine the distance $D_{i s t}$ among all nodes, utilized as an essential quantity of connectivity among the single nodes. We utilized the condition (17), which is expressive the distance among pixels linked to leaf nodes $N D_{i}$ and $N D_{j}$ :

$$
D_{i s t}\left(N D_{i}, N D_{j}\right)=\sqrt{\left(N D_{j} s-N D_{i} s\right)^{2}+\left(N D_{j} t-N_{i} t\right)^{2}}
$$

\section{Building trees from adjoining nodes}

Tree-like organizations area unit made through an algorithmic procedure that considers the gap between the underlying primary leaf nodes. . Algorithm 1 illustrations the overall methodology of building trees from the nodes taking slightest distance. Nearest nodes are combined iteratively to create classification trees

\section{Input: List of nodes \\ Output: Classification tree}

Do iteratively link nodes $\left(N_{o d}\right)$ then
(i). Discovery the nearby $N_{o d}$;
(ii). Eliminating this combine from the $N_{o d}$;
(ii). Join these $N_{o d}$ as a binary tree wherever the $N_{o d}$ square
measure currently delineate as $N_{o d}$ of a binary tree

(iv). Addition the parent of the tree to the $N_{o d}$;

While no more pairs of $N_{o d}$ under a selected space square measure initiate

Return: list of $N_{o d}$, within which nearby $N_{o d}$ are incorporated as trees

Algorithm 1: HOT based classification tree generation

The HOT based classification tree efficiently classifies the breast images into normal and abnormal images. 


\subsection{Classification using HOT based Convolutional neural network (CNN)}

$\mathrm{CNN}$ is demonstrated to have the option to learn significantly improved discriminative graphic depictions for images analogized with early neural networks. Existing CNN [25] can't well handle large-scale image clustering as near typically don't exist adequate labeled data for feature exemplification learning of CNNs. The organization of the CNN classifier is represented in figure 2,

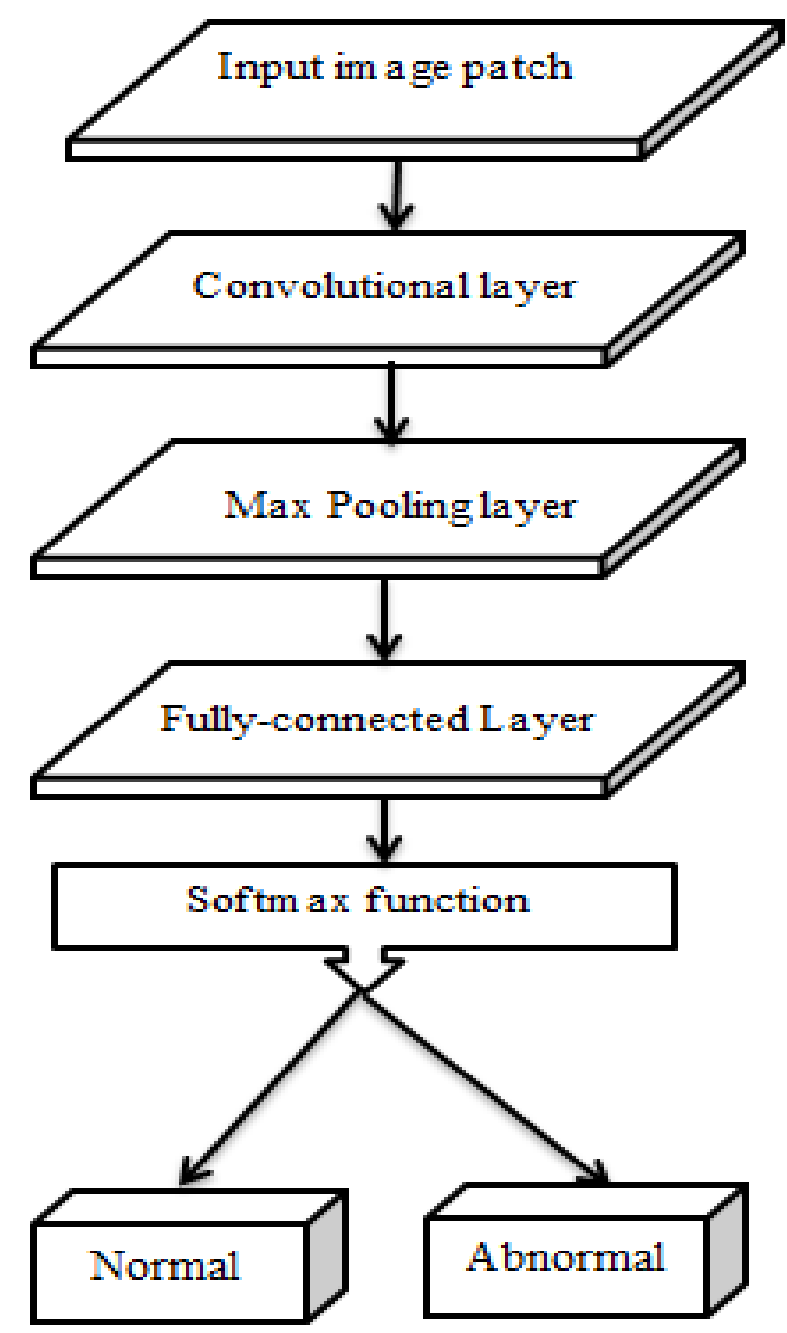

Figure 2: The structure of a convolutional neural network

The CNN concluding resolution depends on the weights and biases of the previous layers in the system design. Thus, the model is rationalized with the condition (18) and condition (19) individually for all layers.

$$
\begin{aligned}
& \Delta W_{l}=-\frac{x \lambda}{r} W_{n}-\frac{x}{N_{t}} \frac{\partial C}{\partial W_{n}}+m \Delta W_{n}(t) \\
& \Delta B_{n}=-\frac{x}{n} \frac{\partial C}{\partial B_{n}}+m \Delta B_{n}(t)
\end{aligned}
$$

Where, $W_{n}$ represents the weight, $B_{n}$ represents the bias, $n$ represents the layer quantity, $\lambda$ represents the regularization factor, $x$ represents the learning level, $N_{t}$ represents the total number of training trials, $m$ represents the energy, $t$ represents the updating step, and $C$ represents the rate function. The CNN includes various kinds of layers are as follows, 
(a) Convolutional layer $\left(C_{o l}\right)$ : In every, initial $C_{o l}$ the yield of past layers is convolved with totally different learned weight networks known as filter masks. This layer moves out the convolution of the computer file with the kernel by exploitation (20).

$$
C_{k}=\sum_{f=0}^{F-1} y_{n} \hat{h}_{k-n}
$$

Where, $y_{n}$ is the feature values, $\hat{h}$ is the filter value, and $F$ is the amount of components in $y$ and the output stored in $C_{k}$.

(b) Pooling layer $\left(P_{o l}\right)$ : The $P_{o l}$ scheme reduces the dimension of resultant neurons from the $C_{o l}$ to scale back the procedure intensity and keep one's eyes off from the over correct. Max $P_{o l}$ activity selects the foremost excessive incentive in each part and on these lines, therefore decreasing the quantity of output neurons. Moreover, the $P_{o l}$ shorten the knowledge within the output from the $C_{o l}$.

(c) Fully connected layer $\left(F_{C l}\right)$ : It contains a complete rapport to each one in every of initiation within the earlier $P_{o l}$. That's this layer interfaces each nerve cell from the utmost $P_{o l}$ to all of the output neurons. The activation perform exploited in this effort is in step with the incidental to,

Softmax ( $S_{\max }$ ): This operate calculates the likelihood distribution of the $F$ output categories. Therefore, the output layer uses the $S_{\max }$ operate to calculate a category input is tailored to normal or abnormal.

$$
p_{i}=\frac{e^{x_{i}}}{\sum_{1}^{k} e^{x_{i}}}
$$

Where, $x$ is the input that is the output classes of CNN are normal and abnormal breast image. Convolution neural networks are concerned with convolution layers that are less challenging to train and they are fundamentally used to group the images, cluster them by similarity, and execute the malignant recognition in scenes.

\section{Results and Discussion}

The proposed breast cancer classification utilizing the proposed HOT based classification tree and CNN was executed in the platform of MATLAB 14 a. The experimental results are examined in two phases are normalabnormal and benign-malignant. The dataset used to evaluate the classification of breast images into normal or abnormal utilizing the proposed characterization tree and CNN classification. The performance of the existing systems is contrasted with the current classifiers with deference to accuracy, sensitivity, specificity, mean absolute error, AUC, kappa statistics and Root mean square error.

\subsection{MIAS Database description}

The Mammographic Image Analysis Society (MIAS) [21] is an association of UK investigation teams involved with the comprehension of mammograms and has created an info of digital mammograms. The dataset has reduced to a 200-micron constituent edge and padded/clipped therefore completely the images are $1024 \times 1024$. The portrayal of the MIAS dataset is given in table1.

Table 1: MIAS dataset description

\begin{tabular}{lll}
\hline \hline & MIAS & \\
\cline { 2 - 3 } & Malignant & Benign \\
\hline \hline Training & 43 & 147 \\
Validation & 9 & 31 \\
Testing & 9 & 31 \\
Overall & 61 & 209 \\
\hline \hline
\end{tabular}




\subsection{Lakeshore Database}

The proposed methodology was simulated on 100 mammographic images from Lakeshore Hospital and the outcomes acquired were confirmed with clinical discoveries of the radiologists. Figure 3 displays the original image, preprocessed image, Gabor image, and histogram images of normal breast image. Along these lines, figure 4 portrays that the original image, preprocessed image, Gabor image, and histogram images of abnormal breast images.

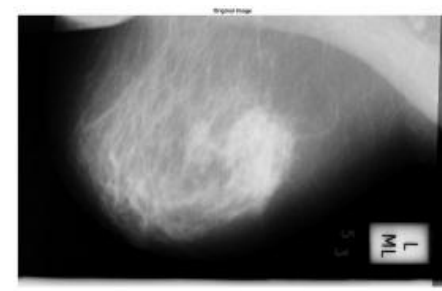

(a) Original Image

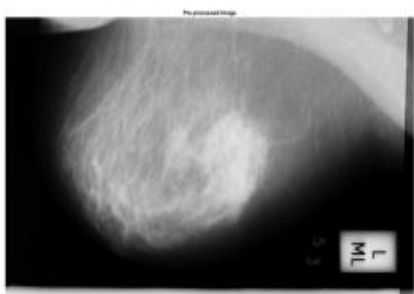

(b) Preprocessed Image
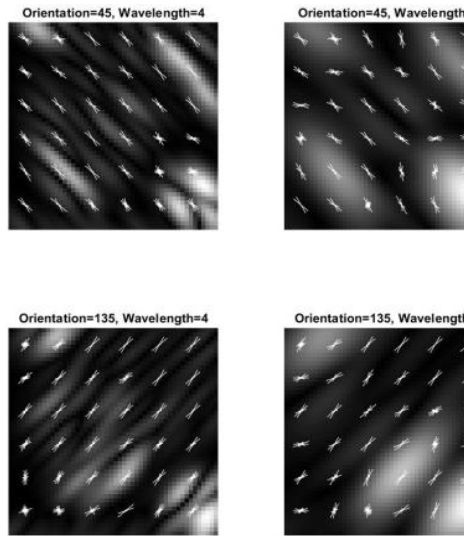

(c) Histogram Image
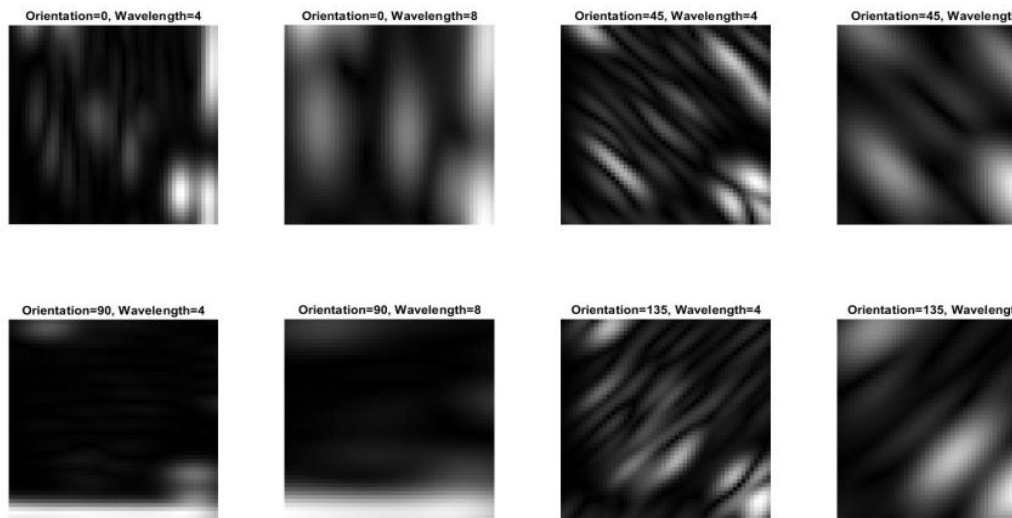

(d) Gabour filter image

Figure 3: Normal image 

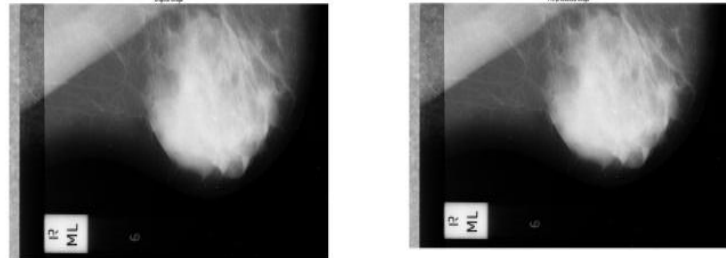

(a) Original Image

(b) Preprocessed Image
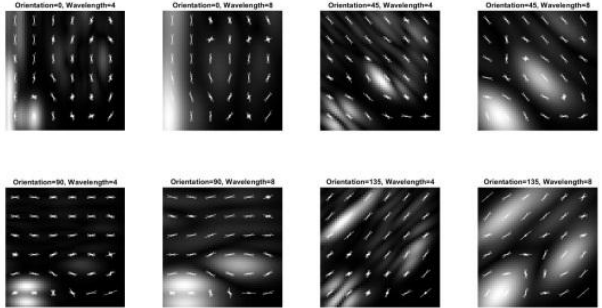

(c) Histogram Image
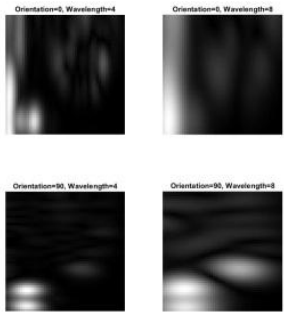

(d) Gabour filter image
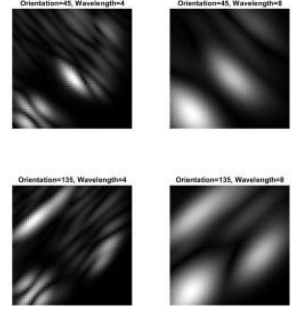
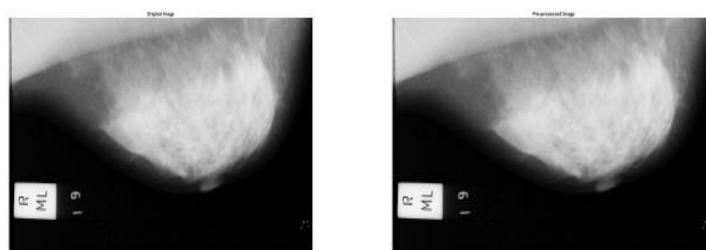

(a) Original Image
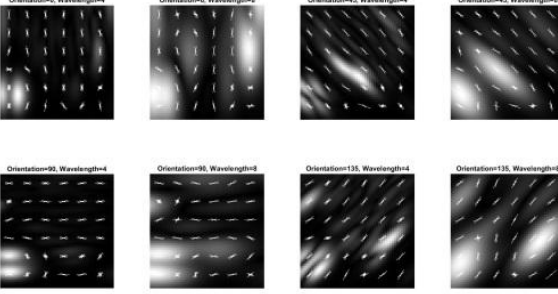

(c) Histogram Image
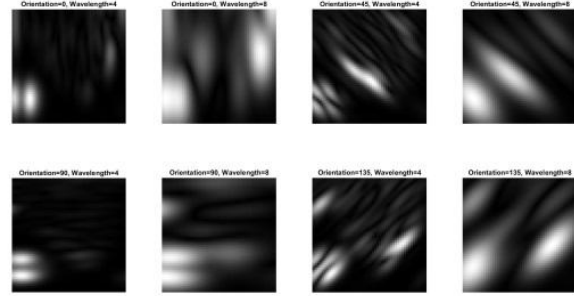

(d) Gabour filter image

Figure 4: Abnormal image

Numerical processes used to confirm the performance of the given work are given within the following sections

\subsection{Performance Analysis}

The numerical metrics of sensitivity, specificity, and accuracy are often represented within the terms of $T_{p o s}$ is True Positive, $F_{p o s}$ is False Positive, $F_{n e g}$ is False Negative, and $T_{n e g}$ is True Negative esteem. The presentation of our proposed work is designed by using the statistical measures mentioned during this section.

\section{$>\operatorname{Accuracy}(A C C)$}

$A C C$ is that the quantity of true effect, either $T_{p o s}$ or $T_{n e g}$, in a very public. It computes the step of exactness of knowledge classification. $A C C$ is definite by using the state (22).

$$
A C C=\frac{\left(T_{\text {neg }}+T_{p o s}\right)}{\left.T_{\text {neg }}+T_{\text {pos }}+F_{\text {neg }}+F_{p o s}\right)}
$$

\section{$>\quad \operatorname{Sensitivity}(\operatorname{SEn})$}

$S E n$ is that the range of $T_{p o s}$ that are with efficiency distinguished by a classification check. It indicates however nice the check is at classifying the information. SEn is computed by utilizing the state (23).

$$
S E n=T_{\text {pos }} /\left(T_{\text {pos }}+F_{\text {neg }}\right)
$$

\section{$>\quad \operatorname{Specificity}(\mathrm{SPe})$}


SPe is the quantity of the $T_{\text {neg }}$ true negatives appropriately recognized by a classification test. It proposed how great the test is at distinguishing normal data. SPe is computed by utilizing the state (24).

$$
S P e=T_{n e g} /\left(T_{n e g}+F_{p o s}\right)
$$

\section{Kappa Statistics value $\left(K_{P a}\right)$}

Measures the chance of an agreement between the predicted and the real classes given by,

$$
K_{P a}=\frac{a \theta_{o}-a \theta_{e}}{1-a \theta_{e}}
$$

Where, $a_{\theta_{o}}$ is the determined agreement, and $a_{\theta_{e}}$ is the predictable agreement.

\section{$>\quad$ Mean Absolute Error $(M A E)$}

MAE expresses the error in classified image. The MAE is computed using state (26).

$$
M A E=\sum_{x=0}^{M} \sum_{y=0}^{N}\left[I^{\left.\left.(x, y)-g^{\prime}(x, y) / n\right)\right]}\right.
$$

Here, $\mathrm{n}$ is the quantity of errors, $I(x, y)-g^{\prime}(x, y)$ absolute errors performance.

\section{Root Mean Square Error ( $R M S E)$}

A small deviation in the examination is identified using the RMSE.

$$
R M S E=\operatorname{sqrt}\left[m\left(o b t_{r e s}-o r i_{. r e s}\right)^{2}\right]
$$

Here, $o b t_{r e s}$ is the attained result calculated from the testing process and $o r i_{\text {.res }}$ is the original result and $\mathrm{m}$ is the mean value. The comparison analysis of the proposed HOT based classification tree (CT) and CNN in phase 1 with existing techniques are given in table 2.

Table 2: Comparative exploration of different classifiers in phase 1

\begin{tabular}{llll}
\hline \hline \multirow{2}{*}{ Method } & MIAS & & \\
\cline { 2 - 4 } & Accuracy & Sensitivity & Specificity \\
\hline \hline GRsca(Nanni et al.,2012) & 86.85 & 68.95 & 98.79 \\
Zernike(Tahmasbi et al.,2011) & 85.39 & 75.92 & 91.70 \\
HOG(Ergin \& Kilinc,2015) & 80.67 & 70 & 87.78 \\
DP-HOT(Shastri et al., 2018) & 93 & 87 & 98 \\
WGLCM(Beura et al.,2015) & 85.89 & 64.72 & 100 \\
Proposed classifier Method1(HOT+CT) & 96.89 & 96.29 & 100 \\
Proposed classifier Method2(HOT+CNN) & 98.5 & 100 & 100 \\
\hline \hline
\end{tabular}

The comparison analysis of sensitivity, specificity, and accuracy for different classifiers-phase 1 is given in figure 5 . 


\section{COMPARATIVE ANALYSIS OF SENSITIVITY, SPECIFICITY AND ACCURACY FOR DIFFERENT CLASSIFIERS- PHASE I}

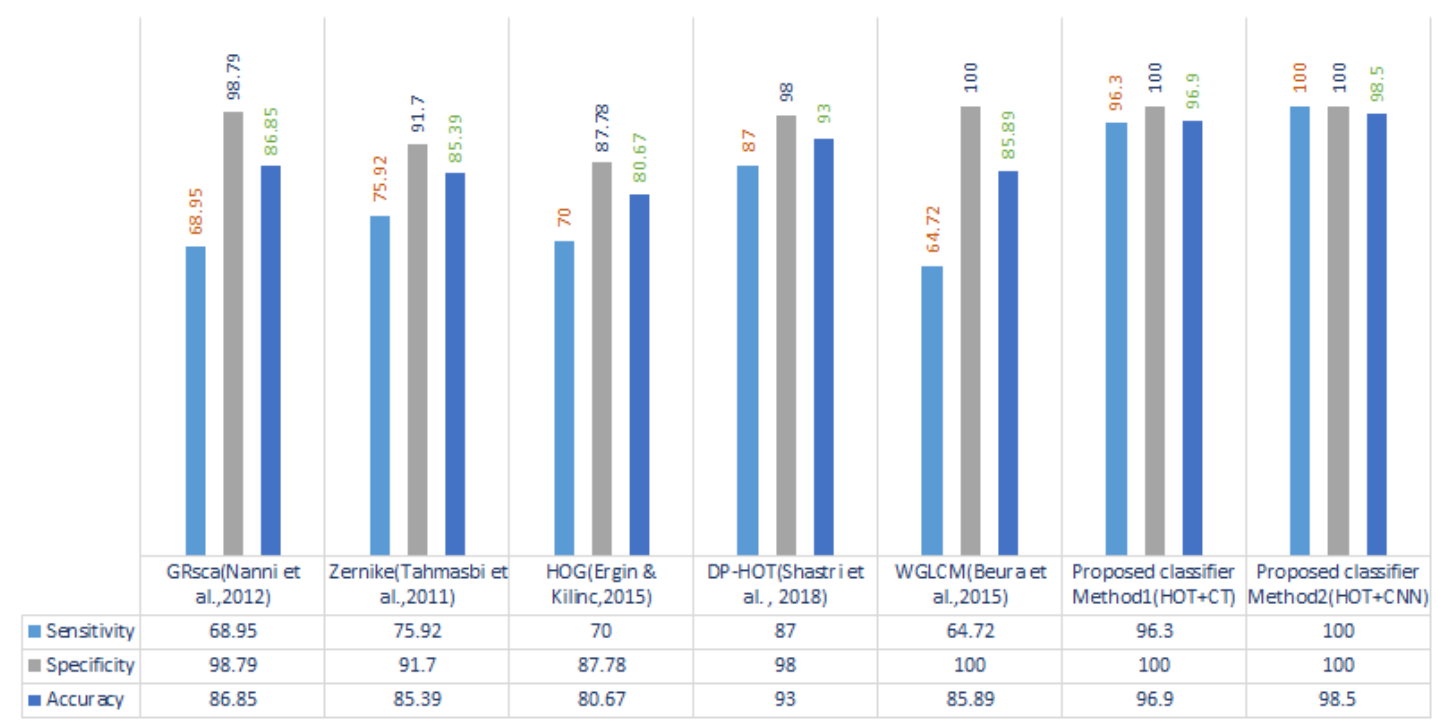

Figure 5: Comparison exploration of sensitivity, specificity, and accuracy for different classifiers-phase 1

The comparison analysis of the proposed HOT based classification tree (CT) and CNN with existing techniques are given in table 3 .

Table 3: Comparative exploration of different classifiers in phase 2

\begin{tabular}{llll}
\hline \hline Method & MIAS & & \\
\cline { 2 - 4 } & Accuracy & Sensitivity & Specificity \\
\hline \hline GRsca(Nanni et al.,2012) & 68.33 & 96.88 & 35.71 \\
Zernike(Tahmasbi et al.,2011) & 82.44 & 80.47 & 84.15 \\
HOG(Ergin \& Kilinc,2015) & 87.5 & 80.67 & 92.86 \\
DP-HOT(Shastri et al., 2018) & 98.24 & 98.83 & 97.57 \\
WGLCM(Beura et al.,2015) & 64.96 & 53.81 & 74.72 \\
Proposed classifier Method1(HOT+CT) & 99.63 & 100 & 98.36 \\
Proposed classifier Method2(HOT+CNN) & 96.27 & 97.01 & 94.52 \\
\hline \hline
\end{tabular}

The comparison analysis of sensitivity, specificity, and accuracy for different classifiers-phase 11 is given in figure 6 . 


\section{COMPARATIVE ANALYSIS OF SENSITIVITY, SPECIFICITY AND ACCURACY FOR DIFFERENT CLASSIFIERS- PHASE II}

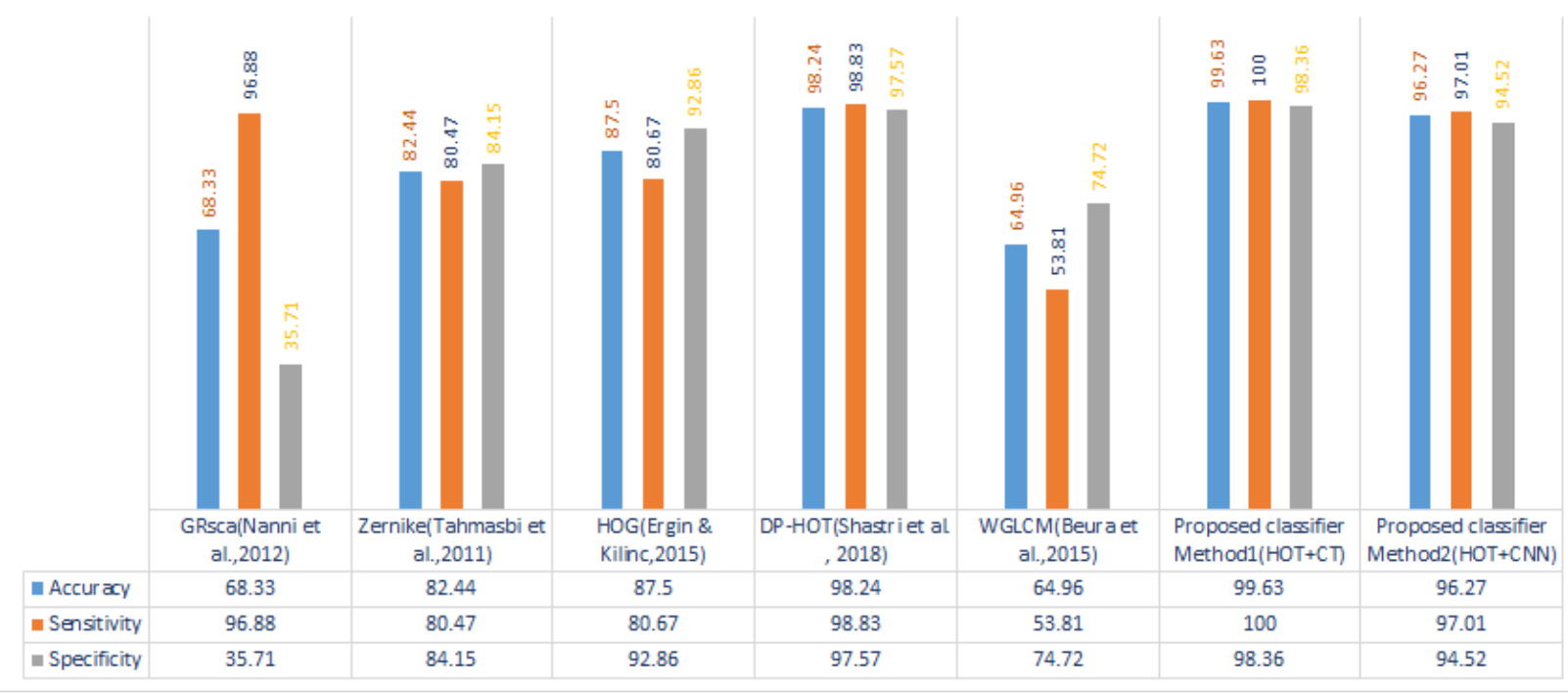

Figure 6: Comparison analysis of sensitivity, specificity, and accuracy for different classifiers-phase 11

The comparison analysis of the proposed HOT based classification tree (CT) and CNN with existing techniques in terms is given in table 4.

Table 4: Accuracy, AUC and standard deviations of different classifiers-phase1 (normal-abnormal)

\begin{tabular}{lll}
\hline \hline Method & Accuracy & AUC \\
\hline \hline GRsca(Nanni et al.,2012) & 86.85 & 91.26 \\
Zernike(Tahmasbi et al.,2011) & 85.39 & 85.11 \\
HOG(Ergin \& Kilinc,2015) & 80.67 & 85.85 \\
DP-HOT(Shastri et al., 2018) & 93 & 97.26 \\
WGLCM(Beura et al.,2015) & 85.89 & 93.41 \\
Proposed classifier Method1(HOT+CT) & 96.89 & 99.14 \\
Proposed classifier Method2(HOT+CNN) & 98.5 & 99.5 \\
\hline \hline
\end{tabular}

The comparison analysis of the proposed HOT based classification tree (CT) and CNN with existing techniques in terms of accuracy and AUC is given in table 5.

Table 5: Accuracies, AUC and standard deviations of different classifiers-phase2 (benign- malignant)

\begin{tabular}{lll}
\hline \hline Method & Accuracy & AUC \\
\hline \hline GRsca(Nanni et al.,2012) & 68.33 & 68.75 \\
Zernike(Tahmasbi et al.,2011) & 82.44 & 72.77 \\
HOG(Ergin \& Kilinc,2015) & 87.5 & 94.20 \\
DP-HOT(Shastri et al. , 2018) & 98.24 & 100 \\
WGLCM(Beura et al.,2015) & 64.96 & 70.09 \\
Proposed classifier Method1(HOT+CT) & 99.63 & 99.97 \\
Proposed classifier Method2(HOT+CNN) & 96.27 & 95.77 \\
\hline \hline
\end{tabular}


The comparison analysis of the proposed HOT based classification tree (CT) and CNN with existing techniques in terms of MAE, RMSE, and Kappa statistic is given in table 6.

Table 6: MAE, RMSE and Kappa Statistic of different classifiers-phase 1

\begin{tabular}{llll}
\hline \hline Method & MIAS & RMSE & $\begin{array}{c}\text { Kappa } \\
\text { Statistic }\end{array}$ \\
\cline { 2 - 4 } & MAE & 0.593 \\
\hline \hline GRsca(Nanni et al.,2012) & & 0.36 & 0.65 \\
Zernike(Tahmasbi et al.,2011) & 0.130 & 0.39 & 0.54 \\
HOG(Ergin \& Kilinc,2015) & 0.15 & 0.44 & 0.84 \\
DP-HOT(Shastri et al., 2018) & 0.27 & 0.67 \\
WGLCM(Beura et al.,2015) & 0.37 & 0.19 \\
Proposed classifier Method1(HOT+CT) & 0.071 & 0.18 & 0.12 \\
Proposed classifier Method2(HOT+CNN) & 0.14 & 0.03 & 0.94 \\
\hline \hline
\end{tabular}

The comparison analysis of MAE, RMSE and Kappa statistics for different classifiers-phase 1 is given in figure 7 .

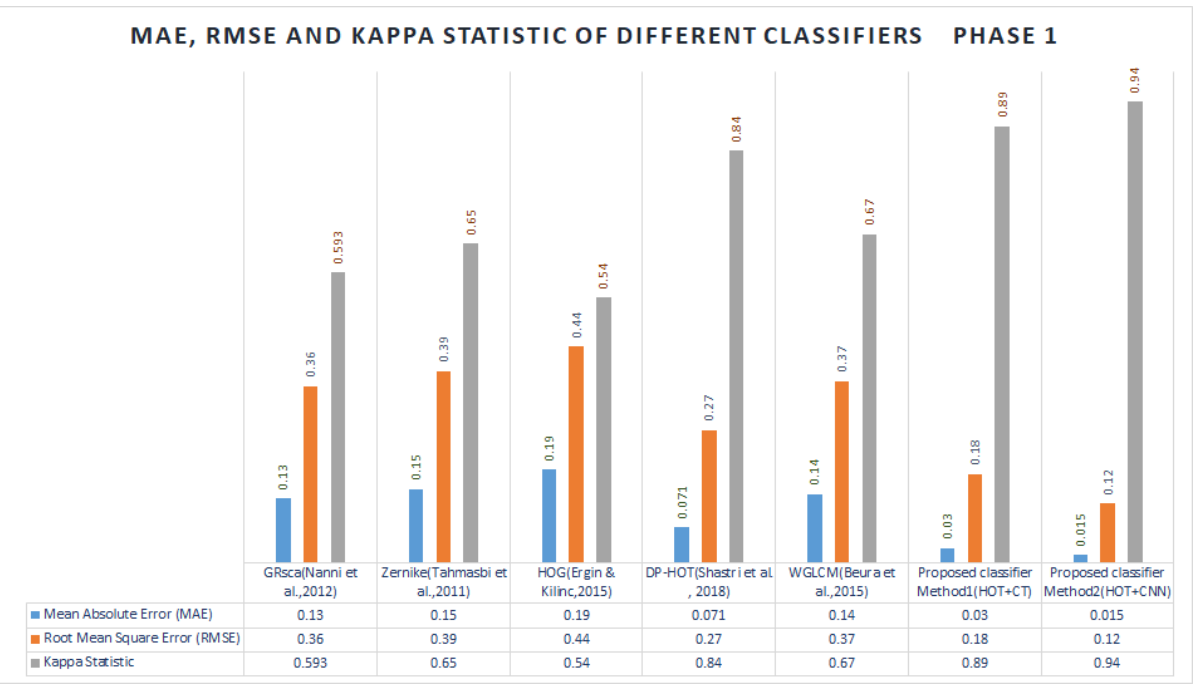

Figure 7: Comparison analysis of sensitivity, specificity, and accuracy for different classifiers-phase 1

The examination results of the proposed methodology in phase 1 and phase 2 with the lakeshore dataset is given in table 7.

Table 7: Examination results of phase 1 and phase 2 with lakeshore dataset

\begin{tabular}{ll|ll}
\hline \hline \multicolumn{2}{l}{ Lakeshore dataset } & \multicolumn{2}{l}{} \\
\hline \hline \multicolumn{2}{l||}{ Phase 1(normal- abnormal) } & \multicolumn{2}{l}{ Phase 2 (benign-malignant) } \\
\hline \hline Specificity & 98.3 & Specificity & 100 \\
Sensitivity & 100 & Sensitivity & 99.45 \\
Accuracy & 98.01 & Accuracy & 98.60 \\
\hline
\end{tabular}

Table 7 proves that the performance of the proposed methodology in phase 11 is improved than phase 1 results. The comparison analysis of the proposed HOT based classification tree (CT) and CNN with existing methods in terms of MAE, RMSE, and Kappa statistic is given in table 8.

Table 8: MAE, RMSE and Kappa Statistic of different classifiers-phase 2

Methods MIAS


MAE

RMSE

Kappa

Statistic

\begin{tabular}{llll} 
GRsca(Nanni et al.,2012) & 0.316 & 0.56 & 0.591 \\
Zernike(Tahmasbi et al.,2011) & 0.18 & 0.42 & 0.61 \\
HOG(Ergin \& Kilinc,2015) & 0.12 & 0.35 & 0.66 \\
DP-HOT(Shastri et al., 2018) & 0.018 & 0.13 & 0.94 \\
WGLCM(Beura et al.,2015) & 0.35 & 0.6 & 0.19 \\
Proposed classifier Method1(HOT+CT) & 0.027 & 0.06 & 0.98 \\
Proposed classifier Method2(HOT+CNN) & 0.0037 & 0.19 & 0.84 \\
\hline
\end{tabular}

The comparison analysis of MAE, RMSE and Kappa statistics for various classifiers-phase 11 is given in figure 8 .

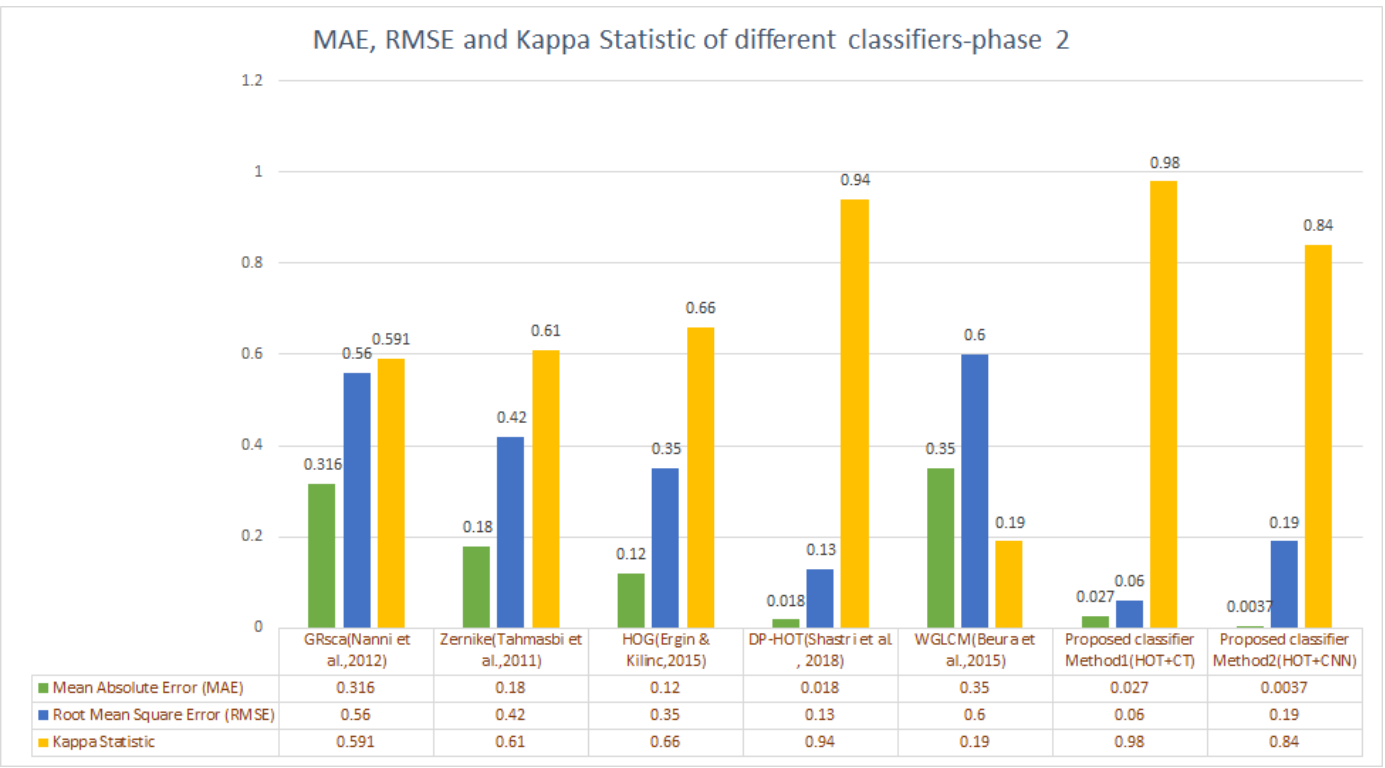

Figure 8: Comparison analysis of sensitivity, specificity, and accuracy for different classifiers-phase 1

Figure 8 shows the proposed HOT based Classification Tree and HOT based CNN gives improved performance than the existing classifications in terms of MAE, RMSE, and kappa statistics.

\section{Conclusion}

In this work, we have presented a successful classification of breast images utilizing HOT based classification tree and HOT based CNN. The input breast image is pre-processed and therefore HOT Descriptor is extracted from the pre-processed images. At last, images are classified as normal or abnormal utilizing HOT based classification tree and HOT based CNN. Here, MIAS and Lakeshore datasets are utilized for the proposed framework analysis. The exploratory outcomes demonstrate that our proposed framework performs viably in breast image classification. The exploratory results show that the introduced framework outperforms the existing methods for various performance analyses like accuracy, sensitivity, specificity, mean absolute error, AUC score, kappa statistics, and Root mean square error

\section{References}

Clarke, Robert, John J. Tyson, and J. Michael Dixon. "Endocrine resistance in breast cancer-an overview and update" Molecular and cellular endocrinology 418 (2015): 220-234.

Bray, Freddie, Jacques Ferlay, Isabelle Soerjomataram, Rebecca L. Siegel, Lindsey A. Torre, and Ahmedin Jemal. "Global cancer statistics 2018: GLOBOCAN estimates of incidence and mortality worldwide for 36 cancers in 185 countries." CA: a cancer journal for clinicians 68, no. 6 (2018): 394-424.

Siu, Albert L. "Screening for breast cancer: US Preventive Services Task Force recommendation statement." Annals of internal medicine 164, no. 4 (2016): 279-296. 
Wuniri, Qiqige, Wei Huangfu, Yaxi Liu, Xiaoli Lin, Liyuan Liu, and Zhigang Yu. "A Generic-Driven Wrapper Embedded With Feature-Type-Aware Hybrid Bayesian Classifier for Breast Cancer Classification." IEEE Access 7 (2019): 119931-119942.

Carroll, Peter R., J. Kellogg Parsons, Gerald Andriole, Robert R. Bahnson, Erik P. Castle, William J. Catalona, Douglas M. Dahl et al. "NCCN guidelines insights: prostate cancer early detection, version 2.2016." Journal of the National Comprehensive Cancer Network 14, no. 5 (2016): 509-519.

Shah, Chirag, Douglas W. Arthur, David Wazer, Atif Khan, Sheila Ridner, and Frank Vicini. "The impact of early detection and intervention of breast cancer-related lymphedema: a systematic review." Cancer medicine 5, no. 6 (2016): 1154-1162.

Moayedi, Fatemeh, Zohreh Azimifar, Reza Boostani, and Serajodin Katebi. "Contourlet-based mammography mass classification using the SVM family" Computers in biology and medicine 40, no. 4 (2010): 373-383.

Wang, Zhentian, Nik Hauser, Gad Singer, Mafalda Trippel, Rahel A. Kubik-Huch, Christof W. Schneider, and Marco Stampanoni. "Non-invasive classification of microcalcifications with phase-contrast X-ray mammography" Nature communications 5, no. 1 (2014): 1-9.

Ponraj, D. Narain, M. Evangelin Jenifer, P. Poongodi, and J. Samuel Manoharan. "A survey on the preprocessing techniques of mammogram for the detection of breast cancer" Journal of Emerging Trends in Computing and Information Sciences 2, no. 12 (2011): 656-664.

Robertson, Stephanie, Hossein Azizpour, Kevin Smith, and Johan Hartman. "Digital image analysis in breast pathology_from image processing techniques to artificial intelligence" Translational Research 194 (2018): 19-35.

Veta, Mitko, Josien PW Pluim, Paul J. Van Diest, and Max A. Viergever. "Breast cancer histopathology image analysis: A review." IEEE Transactions on Biomedical Engineering 61, no. 5 (2014): 1400-1411.

Li, Xingyu, Marko Radulovic, Ksenija Kanjer, and Konstantinos N. Plataniotis. "Discriminative pattern mining for breast cancer histopathology image classification via fully convolutional autoencoder" IEEE Access 7 (2019): 36433-36445.

Gardezi, Syed Jamal Safdar, Ibrahima Faye, Faouzi Adjed, Nidal Kamel, and Muhammad Hussain. "Mammogram classification using chi-square distribution on local binary pattern features." Journal of Medical Imaging and Health Informatics 7, no. 1 (2017): 30-34.

Guo, Zhenhua, Lei Zhang, and David Zhang "A completed modeling of local binary pattern operator for texture classification." IEEE transactions on image processing 19, no. 6 (2010): 1657-1663.

Gardezi, Syed Jamal Safdar, Ibrahima Faye, and Mohamed Meselhy Eltoukhy "Analysis of mammogram images based on texture features of curvelet Sub-bands" In Fifth International Conference on Graphic and Image Processing (ICGIP 2013), vol. 9069, p. 906924, International Society for Optics and Photonics, 2014.

Eltoukhy, Mohamed Meselhy, and Ibrahima Faye "An optimized feature selection method for breast cancer diagnosis in digital mammogram using multiresolution representation" Applied Mathematics \& Information Sciences 8, no. 6 (2014): 2921.

Gardezi, Syed JS, and Ibrahima Faye "Mammogram Classification Based on Morphological Component Analysis (MCA) and Curvelet Decomposition." Neuroscience and Biomedical Engineering 3, no. 1 (2015): 27-33.

AlZubi, Shadi, Naveed Islam, and Maysam Abbod "Multiresolution analysis using wavelet, ridgelet, and curvelet transforms for medical image segmentation" International journal of biomedical imaging 2011 (2011).

Gardezi, Syed Jamal Safdar, Muhammad Awais, Ibrahima Faye, and Fabrice Meriaudeau "Mammogram classification using deep learning features", In 2017 IEEE International Conference on Signal and Image Processing Applications (ICSIPA), pp. 485-488, IEEE, 2017.

Wang, Zhiqiong, Mo Li, Huaxia Wang, Hanyu Jiang, Yudong Yao, Hao Zhang, and Junchang Xin. "Breast cancer detection using extreme learning machine based on feature fusion with CNN deep features." IEEE Access 7 (2019): 105146-105158.

Khan, Hasan Nasir, Ahmad Raza Shahid, Basit Raza, Amir Hanif Dar, and Hani Alquhayz. "Multi-View Feature Fusion Based Four Views Model for Mammogram Classification Using Convolutional Neural Network." IEEE Access 7 (2019): 165724-165733.

Shen, Tianyu, Chao Gou, Jiangong Wang, and Feiyue Wang. "Simultaneous Segmentation and Classification of Mass Region from Mammograms Using a Mixed-Supervision Guided Deep Model" IEEE Signal Processing Letters (2019).

Saha, Monjoy, and Chandan Chakraborty. "Her2net: A deep framework for semantic segmentation and classification of cell membranes and nuclei in breast cancer evaluation." IEEE Transactions on Image Processing 27, no. 5 (2018): 2189-2200.

$\mathrm{Li}$, Yuqian, Junmin Wu, and Qisong Wu. "Classification of breast cancer histology images using multi-size and discriminative patches based on deep learning." IEEE Access 7 (2019): 21400-21408.

Holla, M. Raviraja, and Alwyn R. Pais. "An effective secret image sharing using quantum logic and GPGPU based EDNN super-resolution." Multimedia Tools and Applications (2020): 1-26. 